EUROPEAN JOURNAL OF PURE AND APPLIED MATHEMATICS

Vol. 13, No. 3, 2020, 608-619

ISSN 1307-5543 - www.ejpam.com

Published by New York Business Global

\title{
On the symmetric block design with parameters $(210,77,28)$ admitting a Frobenius group of order 57
}

\author{
Menderes Gashi \\ Department of Mathematics, Faculty of Mathematics and Natural Sciences, \\ University of Prishtina, Avenue "Mother Teresa" 5, 10000 Prishtina, Kosovo
}

\begin{abstract}
In this paper we have proved that for a putative symmetric block design $\mathcal{D}$ with parameters $(210,77,28)$, admitting a Frobenius group $G=\left\langle\rho, \mu \mid \rho^{19}=\mu^{3}=1, \rho^{\mu}=\rho^{7}\right\rangle$ of order 57 , there are exactly six possible orbit structures up to isomorphism with the orbit distribution $[1 ; 19 ; 19 ; 19 ; 19 ; 19 ; 19 ; 19 ; 19 ; 19 ; 19 ; 19]$.
\end{abstract}

2020 Mathematics Subject Classifications: 05B05

Key Words and Phrases: Symmetric block design, Orbit structure, Automorphism group

\section{Introduction and preliminaries}

A $2-(v, k, \lambda)$ design $(\mathcal{P}, \mathcal{B}, I)$ is said to be symmetric if the relation $|\mathcal{P}|=|\mathcal{B}|=v$ holds and in that case we often speak of a symmetric design with parameters $(v, k, \lambda)$. The collection of the parameter sets $(v, k, \lambda)$ for which a symmetric $2-(v, k, \lambda)$ design exists is often called the "spectrum". The determination of the spectrum for symmetric designs is a widely open problem. For example, a finite projective plane of order $n$ is a symmetric design with parameters $\left(n^{2}+n+1, n+1,1\right)$ and it is still unknown whether finite projective planes of non-prime-power order may exist at all.

The existence/non-existence of a symmetric design has often required "ad hoc" treatments even for a single parameter set $(v, k, \lambda)$. The most famous instance of this circumstance is perhaps the non-existence of the projective plane of order 10, see [9].

It is of interest to study symmetric designs with additional properties, which often involve the assumption that a non-trivial automorphism group acts on the design under consideration, see for instance [3].

Among symmetric block designs of square order, a study of symmetric block designs of order 49 is of a particular interest. There are 15 possible parameters $(v, k, \lambda)$ for symmetric designs of order 49, but until now only a few results are known (see [2], [4]). Due to the fact that symmetric designs of order 49 have a big number of points (blocks), the study of

DOI: https://doi.org/10.29020/nybg.ejpam.v13i3.3769

Email addresses: menderes_gashi@yahoo.com (M. Gashi) 
sporadic cases is very difficult, except, possibly, when the existence of a collineation group is assumed.

A few methods for the construction of symmetric designs are known and all of them have shown to be effective in certain situations. Here, we shall use the method of tactical decompositions, assuming that a certain automorphism group acts on the design we want to construct, used by Z. Janko in [7]; see also [3] and [5]. The present paper is concerned with a symmetric design $\mathcal{D}=(\mathcal{P}, \mathcal{B}, I)$ with parameters $(280,63,14)$ : the existence/nonexistence of such a design is still in doubt as far as we know. We shall further assume that the given design admits a certain automorphism group of order 93. We assume the reader is familiar with the basic facts of design theory, see for instance [8], [1] and [10]. If $g$ is an automorphism of a symmetric design $\mathcal{D}$ with parameters $(v, k, \lambda)$, then $g$ fixes an equal number of points and blocks, see [10, Theorem 3.1, p.78]. We denote the sets of these fixed elements by $F_{\mathcal{P}}(g)$ and $F_{\mathcal{B}}(g)$ respectively, and their cardinality simply by $|F(g)|$. We shall make use of the following upper bound for the number of fixed points, see [10, Corollary 3.7, p. 82]:

$$
|F(g)| \leq k+\sqrt{k-\lambda}
$$

It is also known that an automorphism group $G$ of a symmetric design has the same number of orbits on the set of points $\mathcal{P}$ as on the set of blocks $\mathcal{B}$ : [10, Theorem 3.3, p.79]. Denote that number by $t$.

\section{Point- and block-orbits}

We adopt the notation and terminology of Section 1 in [3]. In the following, for the sake of completeness, some fundamental relations are explicitly provided. Let $\mathcal{D}$ be a symmetric design with parameters $(v, k, \lambda)$ and let $G$ be a subgroup of the automorphism group $\operatorname{Aut}(\mathcal{D})$ of $\mathcal{D}$. Denote the point orbits of $G$ on $\mathcal{P}$ by $\mathcal{P}_{1}, \mathcal{P}_{2}, \ldots \mathcal{P}_{t}$ and the line orbits of $G$ on $\mathcal{B}$ by $\mathcal{B}_{1}, \mathcal{B}_{2}, \ldots \mathcal{B}_{t}$. Put $\left|\mathcal{P}_{r}\right|=\omega_{r}$ and $\left|\mathcal{B}_{i}\right|=\Omega_{i}$. Obviously,

$$
\sum_{r=1}^{t} \omega_{r}=\sum_{i=1}^{t} \Omega_{i}=v .
$$

Let $\gamma_{i r}$ be the number of points from $\mathcal{P}_{r}$, which lie on a line from $\mathcal{B}_{i}$; clearly this number does not depend on the chosen line. Similarly, let $\Gamma_{j s}$ be the number of lines from $\mathcal{B}_{j}$ which pass through a point from $\mathcal{P}_{s}$. Then, obviously,

$$
\sum_{r=1}^{t} \gamma_{i r}=k \text { and } \sum_{j=1}^{t} \Gamma_{j s}=k .
$$

By [1, Lemma 5.3.1. p.221], the partition of the point set $\mathcal{P}$ and of the block set $\mathcal{B}$ forms a tactical decomposition of the design $\mathcal{D}$ in the sense of $[1$, p.210]. Thus, the following equations hold: 


$$
\begin{gathered}
\Omega_{i} \cdot \gamma_{i r}=\omega_{r} \cdot \Gamma_{i r} \\
\sum_{r=1}^{t} \gamma_{i r} \Gamma_{j r}=\lambda \Omega_{j}+\delta_{i j}(k-\lambda) \\
\sum_{i=1}^{t} \Gamma_{i r} \gamma_{i s}=\lambda \omega_{s}+\delta_{r s}(k-\lambda)
\end{gathered}
$$

where $\delta_{i j}, \delta_{r s}$ are the Kronecker symbols.

For a proof of these equations, the reader is referred to [1] and [3]. Equation (5), together with (4) yields

$$
\sum_{r=1}^{t} \frac{\Omega_{j}}{\omega_{r}} \gamma_{i r} \gamma_{j r}=\lambda \Omega_{j}+\delta_{i j}(k-\lambda) .
$$

Definition 1. We denote

$$
\left[L_{i}, L_{j}\right]=\sum_{r=1}^{t} \frac{\Omega_{j}}{\omega_{r}} \gamma_{i r} \gamma_{j r}, 1 \leq i, j \leq t
$$

and call these expressions the orbit products. The $(t \times t)$-matrix $\left(\gamma_{i r}\right)$ is called the orbit structure of the design $\mathcal{D}$.

An automorphism of an orbit structure is a permutation of rows followed by a permutation of columns leaving that matrix unchanged. It is clear that the set of all such automorphisms is a group, which we call the automorphism group of that orbit structure.

The first step in the construction of a design is to find all possible orbit structures. The second step of the construction is usually called indexing. In fact for each coefficient $\gamma_{i r}$ of the orbit matrix one has to specify which $\gamma_{i r}$ points of the point orbit $\mathcal{P}_{r}$ lie on the lines of the block orbit $\mathcal{B}_{i}$. Of course, it is enough to do this for a representative of each block orbit, as the other lines of that orbit can be obtained by producing all $G$-images of the given representative.

\section{Action of the Frobenius group of order 57}

In our construction of symmetric $2-(210,77,28)$ designs we assume the existence of an automorphism group $G=\left\langle\rho, \mu \mid \rho^{19}=\mu^{3}=1, \rho^{\mu}=\rho^{7}\right\rangle$, which is a so called Frobenius group of order 57 with Frobenius kernel of order 19 (see [6]).

Theorem 1. Up to isomorphism there are exactly six orbit structures for symmetric (210,77,28) designs and the automorphism group $G=F_{19 \cdot 3}$ acting with the orbit distribution $\mathcal{O}=[1 ; 19 ; 19 ; 19 ; 19 ; 19 ; 19 ; 19 ; 19 ; 19 ; 19 ; 19]$. 
Proof. We denote by $\infty$ the fixed point of $\rho$ and put $\mathcal{P}_{I}=\left\{I_{0}, I_{1}, \cdots, I_{18}\right\}, I=$ $1,2,3,4,5,6,7,8,9,10,11$, for the non-trivial orbits of the group $G$. Thus, $G$ acts on these point orbits as a permutation group in a unique way. Hence, for the two generators of $G$ we may put

$$
\rho=(\infty)\left(I_{0}, I_{1}, \cdots, I_{18}\right), I=1,2, \cdots, 11,
$$

where $\infty$ is the fixed point of collineation, whereas non-trivial $\langle\rho\rangle$-orbits are numbers $1,2,3,4,5,6,7,8,9,10,11$ and $\infty, 1_{0}, 1_{1}, \cdots, 11_{18}$ all points of the symmetric block design $\mathcal{D}$, and the collineation $\mu$ of order 3 acts in the symmetric block design as permutation $(1)(2)(3)(4)(5)(6)(7)(8)(9)(10)(11)$ on orbit numbers, whereas on indices acts $\mu: x \rightarrow 7 x$ $(\bmod 19)$ or

$$
\begin{gathered}
\mu=(\infty)\left(K_{0}\right)\left(K_{1}, K_{7}, K_{11}\right)\left(K_{2}, K_{14}, K_{3}\right)\left(K_{4}, K_{9}, K_{6}\right)\left(K_{5}, K_{16}, K_{17}\right) \\
\left(K_{8}, K_{18}, K_{12}\right)\left(K_{10}, K_{13}, K_{15}\right), \quad K=1,2,3,4,5,6,7,8,9,10,11 .
\end{gathered}
$$

We immediately obtain the following.

Corollary 1. The element $\mu$ of $G$ of order 3 fixes precisely 12 points and 12 blocks of $\mathcal{D}$. Each block orbit contains a unique block stabilized by $\mu$.

In what follows, we are going to construct a representative block for each block orbit. A representative block for the block orbit of length 19 will be the block fixed by $\mu$. Hence the multiplicities of orbit numbers in orbit blocks, will be $\equiv 0,1(\bmod 3)$.

The $\langle\rho\rangle$-fixed block can be writen in the form:

$$
L_{1}=\infty\left(1_{0} 1_{1} \cdots 1_{18}\right)\left(2_{0} 2_{1} \cdots 2_{18}\right)\left(3_{0} 3_{1} \cdots 3_{18}\right)\left(4_{0} 4_{1} \cdots 4_{18}\right)
$$

or

$$
L_{1}=\infty 1_{19} 2_{19} 3_{19} 4_{19} .
$$

Let $L_{2}, L_{3}, L_{4}, L_{5}, L_{6}, L_{7}, L_{8}, L_{9}, L_{10}, L_{11}, L_{12}$ be the representative blocks for the eleven non-trivial block orbits. There are exactly four non-fixed orbit blocks passing through the fixed point $\infty$. Let them be $L_{2}, L_{3}, L_{4}, L_{5}$. We write

$$
\begin{aligned}
L_{2} & =\infty 1_{a_{1}} 2_{a_{2}} 3_{a_{3}} 4_{a_{4}} 5_{a_{5}} 6_{a_{6}} 7_{a_{7}} 8_{a_{8}} 9_{a_{9}} 10_{a_{10}} 11_{a_{11}} \\
L_{3} & =\infty 1_{b_{1}} 2_{b_{2}} 3_{b_{3}} 4_{b_{4}} 5_{b_{5}} 6_{b_{6}} 7_{b_{7}} 8_{b_{8}} 9_{b_{9}} 10_{b_{10}} 11_{b_{11}} \\
L_{4} & =\infty 1_{c_{1}} 2_{c_{2}} 3_{c_{3}} 4_{c_{4}} 5_{c_{5}} 6_{c_{6}} 7_{c_{7}} 8_{c_{8}} 9_{c_{9}} 10_{c_{10}} 11_{c_{11}} \\
L_{5} & =\infty 1_{d_{1}} 2_{d_{2}} 3_{d_{3}} 4_{d_{4}} 5_{d_{5}} 6_{d_{6}} 7_{d_{7}} 8_{d_{8}} 9_{d_{9}} 10_{d_{10}} 11_{d_{11}}
\end{aligned}
$$

where $a_{i}, b_{i}, c_{i}, d_{i}$ denote the multiplicities of the appearance of orbit numbers in the orbit blocks $L_{2}, L_{3}, L_{4}, L_{5}$, respectively.

The multiplicities of the appearances of orbit numbers satisfy the following conditions: 


$$
\begin{gathered}
a_{1}+a_{2}+a_{3}+a_{4}+a_{5}+a_{6}+a_{7}+a_{8}+a_{9}+a_{10}+a_{11}=76 . \\
b_{1}+b_{2}+b_{3}+b_{4}+b_{5}+b_{6}+b_{7}+b_{8}+b_{9}+b_{10}+b_{11}=76 . \\
c_{1}+c_{2}+c_{3}+c_{4}+c_{5}+c_{6}+c_{7}+c_{8}+c_{9}+c_{10}+c_{11}=76 . \\
d_{1}+d_{2}+d_{3}+d_{4}+d_{5}+d_{6}+d_{7}+d_{8}+d_{9}+d_{10}+d_{11}=76 .
\end{gathered}
$$

Because $\left|L_{i} \cap L_{1}\right|=28, i=2,3,4,5$ and $\infty \in L_{i}, i=1,2,3,4,5$ we have $a_{1}+a_{2}+$ $a_{3}+a_{4}=27, b_{1}+b_{2}+b_{3}+b_{4}=27, c_{1}+c_{2}+c_{3}+c_{4}=27, d_{1}+d_{2}+d_{3}+d_{4}=27$ and consequently $a_{5}+a_{6}+a_{7}+a_{8}+a_{9}+a_{10}+a_{11}=49, b_{5}+b_{6}+b_{7}+b_{8}+b_{9}+b_{10}+b_{11}=49$, $c_{5}+c_{6}+c_{7}+c_{8}+c_{9}+c_{10}+c_{11}=49, d_{5}+d_{6}+d_{7}+d_{8}+d_{9}+d_{10}+d_{11}=49$. From (7) we have

$$
\begin{aligned}
& {\left[L_{2}, L_{2}\right]=19 / 1 \cdot 1 \cdot 1+19 / 19 \cdot a_{1}^{2}+19 / 19 \cdot a_{2}^{2}+19 / 19 \cdot a_{3}^{2}+19 / 19 \cdot a_{4}^{2}+19 / 19 \cdot a_{5}^{2}+19 / 19 \cdot a_{6}^{2}} \\
& +19 / 19 \cdot a_{7}^{2}+19 / 19 \cdot a_{8}^{2}+19 / 19 \cdot a_{9}^{2}+19 / 19 \cdot a_{10}^{2}+19 / 19 \cdot a_{11}^{2}=28 \cdot 19+77-28=581 \\
& {\left[L_{3}, L_{3}\right]=19 / 1 \cdot 1 \cdot 1+19 / 19 \cdot b_{1}^{2}+19 / 19 \cdot b_{2}^{2}+19 / 19 \cdot b_{3}^{2}+19 / 19 \cdot b_{4}^{2}+19 / 19 \cdot b_{5}^{2}+19 / 19 \cdot b_{6}^{2}} \\
& +19 / 19 \cdot b_{7}^{2}+19 / 19 \cdot b_{8}^{2}+19 / 19 \cdot b_{9}^{2}+19 / 19 \cdot b_{10}^{2}+19 / 19 \cdot b_{11}^{2}=28 \cdot 19+77-28=581 \\
& {\left[L_{4}, L_{4}\right]=19 / 1 \cdot 1 \cdot 1+19 / 19 \cdot c_{1}^{2}+19 / 19 \cdot c_{2}^{2}+19 / 19 \cdot c_{3}^{2}+19 / 19 \cdot c_{4}^{2}+19 / 19 \cdot c_{5}^{2}+19 / 19 \cdot c_{6}^{2}} \\
& +19 / 19 \cdot c_{7}^{2}+19 / 19 \cdot c_{8}^{2}+19 / 19 \cdot c_{9}^{2}+19 / 19 \cdot c_{10}^{2}+19 / 19 \cdot c_{11}^{2}=28 \cdot 19+77-28=581 \\
& {\left[L_{5}, L_{5}\right]=19 / 1 \cdot 1 \cdot 1+19 / 19 \cdot d_{1}^{2}+19 / 19 \cdot d_{2}^{2}+19 / 19 \cdot d_{3}^{2}+19 / 19 \cdot d_{4}^{2}+19 / 19 \cdot d_{5}^{2}+19 / 19 \cdot d_{6}^{2}} \\
& +19 / 19 \cdot d_{7}^{2}+19 / 19 \cdot d_{8}^{2}+19 / 19 \cdot d_{9}^{2}+19 / 19 \cdot d_{10}^{2}+19 / 19 \cdot d_{11}^{2}=28 \cdot 19+77-28=581 \\
& {\left[L_{2}, L_{3}\right]=19 \cdot 1 \cdot 1+19 / 19 \cdot a_{1} b_{1}+19 / 19 \cdot a_{2} b_{2}+19 / 19 \cdot a_{3} b_{3}+19 / 19 \cdot a_{4} b_{4}+19 / 19 \cdot a_{5} b_{5}} \\
& +19 / 19 \cdot a_{6} b_{6}+19 / 19 \cdot a_{7} b_{7}+19 / 19 \cdot a_{8} b_{8}+19 / 19 \cdot a_{9} b_{9}+19 / 19 \cdot a_{10} b_{10}+19 / 19 \cdot a_{11} b_{11}=28 \cdot 19=532 \\
& {\left[L_{2}, L_{4}\right]=19 \cdot 1 \cdot 1+19 / 19 \cdot a_{1} c_{1}+19 / 19 \cdot a_{2} c_{2}+19 / 19 \cdot a_{3} c_{3}+19 / 19 \cdot a_{4} c_{4}+19 / 19 \cdot a_{5} c_{5}} \\
& +19 / 19 \cdot a_{6} c_{6}+19 / 19 \cdot a_{7} c_{7}+19 / 19 \cdot a_{8} c_{8}+19 / 19 \cdot a_{9} c_{9}+19 / 19 \cdot a_{10} c_{10}+19 / 19 \cdot a_{11} c_{11}=28 \cdot 19=532 \\
& {\left[L_{2}, L_{5}\right]=19 \cdot 1 \cdot 1+19 / 19 \cdot a_{1} d_{1}+19 / 19 \cdot a_{2} d_{2}+19 / 19 \cdot a_{3} d_{3}+19 / 19 \cdot a_{4} d_{4}+19 / 19 \cdot a_{5} d_{5}} \\
& +19 / 19 \cdot a_{6} d_{6}+19 / 19 \cdot a_{7} d_{7}+19 / 19 \cdot a_{8} d_{8}+19 / 19 \cdot a_{9} d_{9}+19 / 19 \cdot a_{10} d_{10}+19 / 19 \cdot a_{11} d_{11}=28 \cdot 19=532
\end{aligned}
$$$$
\text { where } 0 \leq a_{i} \leq 23, i=1,2, \cdots, 11 \text {. }
$$

In order to reduce isomorphic cases that may appear in the orbit structures at the last stage, without loss of generality, for the block $L_{2}$, we can use the reduction $a_{1} \geq a_{2} \geq$ $a_{3} \geq a_{4}, a_{5} \geq a_{6} \geq a_{7} \geq a_{8} \geq a_{9} \geq a_{10} \geq a_{11}$.

Using the computer we have proved that there exist exactly twenty one different orbit types for the block $L_{2}$ satisfying the above mentioned conditions: 


\begin{tabular}{|c|c|c|c|c|c|c|c|c|c|c|}
\hline$a_{1}$ & $a_{2}$ & $a_{3}$ & $a_{4}$ & $a_{5}$ & $a_{6}$ & $a_{7}$ & $a_{8}$ & $a_{9}$ & $a_{19}$ & $a_{1}$ \\
\hline 10 & 9 & 4 & 4 & 9 & 7 & 7 & 7 & 7 & 6 & $c$ \\
\hline 10 & 7 & 7 & 3 & 10 & 7 & 7 & 7 & 6 & $c$ & \\
\hline 10 & 7 & 7 & 3 & 9 & 9 & 7 & $\rho$ & 6 & o & \\
\hline 10 & 7 & 6 & 4 & 10 & 9 & 6 & U & U & o & \\
\hline 10 & 7 & 6 & 4 & 10 & 7 & 7 & 7 & $r$ & 7 & \\
\hline 10 & 7 & 6 & 4 & 9 & 9 & 7 & 7 & 7 & 6 & \\
\hline 7. & 9 & 6 & 3 & 10 & 7 & 7 & 7 & 6 & 6 & \\
\hline 8. & 9 & 6 & 3 & 9 & 9 & 7 & 6 & 6 & 6 & \\
\hline 9. & 7 & 7 & 4 & 10 & 9 & 7 & 7 & 6 & 6 & \\
\hline 9 & 7 & 7 & 4 & 9 & 9 & 9 & U & 6 & 6 & \\
\hline 9 & 7 & 7 & 4 & 9 & 9 & 7 & 7 & 7 & 7 & \\
\hline 9 & 6 & 6 & 6 & 12 & 7 & 6 & 6 & 0 & 6 & \\
\hline 9 & 6 & 6 & 6 & 10 & 10 & 7 & 0 & 6 & 6 & \\
\hline 9 & 6 & 6 & 6 & 10 & 9 & 7 & 7 & 7 & 6 & \\
\hline 5. & 6 & 6 & 6 & 9 & 9 & 9 & 7 & 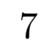 & 4 & \\
\hline 6. & 6 & 6 & 6 & 9 & 9 & 9 & 7 & 6 & 6 & \\
\hline 7 & 7 & 7 & 6 & 12 & 7 & 7 & $r$ & 6 & 6 & \\
\hline 7 & 7 & 7 & 6 & 10 & 10 & 7 & 7 & 7 & 4 & \\
\hline 7 & 7 & 7 & 6 & 10 & 10 & 7 & . & 0 & 6 & \\
\hline 7 & 7 & 7 & 6 & 10 & 9 & 9 & 7 & 6 & 4 & \\
\hline 7 & 7 & 7 & 6 & 10 & 9 & 9 & 6 & 6 & 6 & \\
\hline
\end{tabular}

Further on, we find the possible candidates for $L_{3}$ considering each orbit type for $L_{2}$. Among the candidates for block $L_{3}$ are blocks $L_{4}, L_{5}$. Therefore, for each case for $L_{2}$, from the candidates for $L_{3}$, must be found triples of blocks $\left\{L_{3}, L_{4}, L_{5}\right\}$, which are pairwise compatibile.

Using the computer we obtain the results which we present in the table below: 
Table 1:

\begin{tabular}{|l|c|c|}
\hline Block $L_{2}$ & $\begin{array}{c}\text { Number of orbit } \\
\text { types for } L_{3}\end{array}$ & $\begin{array}{c}\text { Number of quadru- } \\
\text { ples }\left\{L_{2}, L_{3}, L_{4}, L_{5}\right\}\end{array}$ \\
\hline Type 1. & 969 & 8 \\
\hline Type 2. & 1201 & 15 \\
\hline Type 3. & 2073 & 41 \\
\hline Type 4. & 1847 & 13 \\
\hline Type 5. & 2355 & 6 \\
\hline Type 6. & 1172 & 57 \\
\hline Type 7. & 1401 & 8 \\
\hline Type 8. & 1792 & 21 \\
\hline Type 9. & 1001 & 67 \\
\hline Type 10. & 2001 & 43 \\
\hline Type 11. & 1301 & 23 \\
\hline Type 12. & 1170 & 0 \\
\hline Type 13. & 1371 & 11 \\
\hline Type 14. & 1295 & 35 \\
\hline Type 15. & 1584 & 8 \\
\hline Type 16. & 1902 & 55 \\
\hline Type 17. & 1001 & 11 \\
\hline Type 18. & 2340 & 13 \\
\hline Type 19. & 1026 & 28 \\
\hline Type 20. & 1131 & 46 \\
\hline Type 21. & 2098 & 69 \\
\hline
\end{tabular}

Therefore, there are 578 quadruples $L_{2}, L_{3}, L_{4}, L_{5}$ (i.e. 578 quintuples $L_{1}, L_{2}, L_{3}, L_{4}, L_{5}$ ). The sixth orbit block $L_{6}$ has the form:

$$
L_{6}=1_{e_{1}} 2_{e_{2}} 3_{e_{3}} 4_{e_{4}} 5_{e_{5}} 6_{e_{6}} 7_{e_{7}} 8_{e_{8}} 9_{e_{9}} 10_{e_{10}} 11 e_{11}
$$

where $e_{i}, i=1,2, \cdots, 11$ are multiplicities of the appearance of orbit numbers $1,2,3$, $4,5,6,7,8,9,10$ and 11 in orbit block $L_{6}$.

We have: $e_{1}+e_{2}+e_{3}+e_{4}+e_{5}+e_{6}+e_{7}+e_{8}+e_{9}+e_{10}+e_{11}=77$,

$$
\begin{gathered}
{\left[L_{6}, L_{6}\right]=e_{1}^{2}+e_{2}^{2}+e_{3}^{2}+e_{4}^{2}+e_{5}^{2}+e_{6}^{2}+e_{7}^{2}+e_{8}^{2}+e_{9}^{2}+e_{10}^{2}+e_{11}^{2}=28 \cdot 19+77-28=581,} \\
{\left[L_{6}, L_{i}\right]=28 \cdot 19=532,(i=2,3,4,5) .}
\end{gathered}
$$

$\left[L_{6} \cap L_{1}\right]=28$ implies $e_{1}+e_{2}+e_{3}+e_{4}=28$, therefore $c_{5}+c_{6}+c_{7}+c_{8}+c_{9}+c_{10}+c_{11}=$ $77-28=49$.

$\left[L_{6}, L_{6}\right]=581$ implies $0 \leq c_{i} \leq 24, i=1,2, \cdots, 11$. 
Candidates for $L_{6}$ are also blocks $L_{7}, L_{8}, L_{9}, L_{10}, L_{11}$, and $L_{12}$, so we look for septuples $\left\{L_{6}, L_{7}, L_{8}, L_{9}, L_{10}, L_{11}, L_{12}\right\}$ which are pairwise compatibile. Using the computer for the number of quadruples $\left\{L_{2}, L_{3}, L_{4}, L_{5}\right\}$ given in Table 1 , we obtain the results which we present in the table below:

\begin{tabular}{|c|c|c|c|}
\hline $\begin{array}{l}\text { Type } \\
\text { for } L_{2}\end{array}$ & $\begin{array}{l}\text { Subcase for } \\
\text { quadruples } \\
\left\{L_{2}, L_{3}, L_{4}, L_{5}\right\}\end{array}$ & $\begin{array}{l}\text { Number of or- } \\
\text { bit types for } \\
L_{6}\end{array}$ & $\begin{array}{l}\text { Number of septuples } \\
\left\{L_{6}, L_{7}, L_{8}, L_{9}, L_{10}, L_{11}, L_{12}\right\}\end{array}$ \\
\hline 1 & 1 & 48 & 0 \\
\hline 1 & 2 & 48 & 0 \\
\hline 2 & 3 & 40 & 0 \\
\hline 2 & 4 & 40 & 0 \\
\hline 2 & 6 & 60 & 0 \\
\hline 2 & 8 & 60 & 0 \\
\hline 3 & 3 & 32 & 0 \\
\hline 3 & 4 & 22 & 0 \\
\hline 3 & 12 & 78 & 0 \\
\hline 3 & 13 & 33 & 0 \\
\hline 3 & 14 & 20 & 0 \\
\hline 3 & 15 & 23 & 0 \\
\hline 3 & 16 & 23 & 0 \\
\hline 3 & 18 & 33 & 0 \\
\hline 3 & 19 & 79 & 0 \\
\hline 5 & 1 & 120 & 12 \\
\hline 5 & 6 & 48 & 0 \\
\hline 6 & 42 & 48 & 0 \\
\hline 7 & 8 & 25 & 0 \\
\hline 9 & 22 & 22 & 0 \\
\hline 9 & 59 & 7 & 1 \\
\hline 9 & 60 & 22 & 1 \\
\hline 9 & 63 & 36 & 0 \\
\hline 10 & 41 & 45 & 4 \\
\hline 11 & 1 & 48 & 0 \\
\hline 11 & 2 & 48 & 0 \\
\hline 11 & 10 & 32 & 0 \\
\hline 11 & 17 & 39 & 0 \\
\hline 13 & 7 & 45 & 4 \\
\hline 13 & 10 & 32 & 0 \\
\hline 13 & 11 & 39 & 0 \\
\hline 14 & 4 & 25 & 0 \\
\hline 15 & 3 & 22 & 1 \\
\hline 15 & 5 & 25 & 0 \\
\hline 15 & 6 & 25 & 0 \\
\hline 15 & 7 & 36 & 0 \\
\hline 15 & 8 & 36 & 0 \\
\hline 16 & 23 & 36 & 0 \\
\hline 16 & 24 & 7 & 1 \\
\hline 16 & 29 & 39 & 0 \\
\hline 16 & 54 & 74 & 0 \\
\hline 16 & 55 & 74 & 0 \\
\hline
\end{tabular}




\begin{tabular}{|c|c|c|c|}
\hline $\begin{array}{l}\text { Type } \\
\text { for } L_{2}\end{array}$ & $\begin{array}{l}\text { Subcase for } \\
\text { quadruples } \\
\left\{L_{2}, L_{3}, L_{4}, L_{5}\right\}\end{array}$ & $\begin{array}{l}\text { Number of or- } \\
\text { bit types for } L_{6}\end{array}$ & $\begin{array}{l}\text { Number of septuples } \\
\left\{L_{6}, L_{7}, L_{8}, L_{9}, L_{10}, L_{11}, L_{12}\right\}\end{array}$ \\
\hline 17 & 3 & 60 & 0 \\
\hline 17 & 5 & 23 & 0 \\
\hline 17 & 11 & 36 & 0 \\
\hline 18 & 1 & 40 & 0 \\
\hline 18 & 2 & 78 & 0 \\
\hline 18 & 3 & 60 & 0 \\
\hline 19 & 5 & 33 & 0 \\
\hline 19 & 6 & 20 & 0 \\
\hline 19 & 15 & 22 & 1 \\
\hline 19 & 20 & 32 & 0 \\
\hline 19 & 21 & 36 & 0 \\
\hline 19 & 22 & 22 & 1 \\
\hline 19 & 23 & 32 & 0 \\
\hline 19 & 26 & 74 & 0 \\
\hline 19 & 27 & 36 & 0 \\
\hline 20 & 7 & 40 & 0 \\
\hline 20 & 9 & 33 & 0 \\
\hline 20 & 10 & 23 & 0 \\
\hline 20 & 34 & 32 & 0 \\
\hline 21 & 11 & 78 & 0 \\
\hline 21 & 43 & 36 & 0 \\
\hline 21 & 45 & 7 & 1 \\
\hline 21 & 46 & 39 & 0 \\
\hline 21 & 47 & 45 & 4 \\
\hline 21 & 48 & 7 & 1 \\
\hline 21 & 56 & 39 & 0 \\
\hline 21 & 57 & 7 & 1 \\
\hline 21 & 59 & 39 & 0 \\
\hline 21 & 61 & 7 & 1 \\
\hline 21 & 63 & 74 & 0 \\
\hline 21 & 64 & 39 & 0 \\
\hline 21 & 65 & 45 & 4 \\
\hline \multirow[t]{2}{*}{21} & 66 & 36 & 0 \\
\hline & Other subcases & $<7$ & 0 \\
\hline
\end{tabular}

From the table above it can be seen that there are 75 subcases, for which the number of orbit types for $L_{6}$ is greater than 6 , and for those subcases we have searched for septuples $\left\{L_{6}, L_{7}, L_{8}, L_{9}, L_{10}, L_{11}, L_{12}\right\}$, respectively, we have searched for orbit structures. For these 75 subcases we have found 38 orbit structures. After the removal of the isomorphic cases, there remained exactly 6 orbit structures, which are given below: 
Table 2:

\begin{tabular}{|c|c|c|c|c|c|c|c|c|c|c|c|c|c|c|c|c|c|c|c|c|c|c|c|}
\hline $\mathrm{S} 1$. & 19 & & & & & & & & & & & $\mathrm{~S} 2$ & & & & & & & & & & & \\
\hline & $\begin{array}{ll}119 \\
\end{array}$ & 919 & 19 & 19 & 0 & 0 & 0 & 0 & 0 & 0 & $\overline{0}$ & & $\begin{array}{lll}1 & 19\end{array}$ & 19 & 19 & 19 & 0 & 0 & 0 & 0 & 0 & 0 & 0 \\
\hline & 110 & 7 & 6 & 4 & 10 & 7 & 7 & 7 & 7 & 7 & 4 & & 110 & 7 & 6 & 4 & 10 & 7 & 7 & 7 & 7 & 7 & 4 \\
\hline & 17 & 10 & 4 & 6 & 4 & 7 & 7 & 7 & 7 & 7 & 10 & & 17 & 10 & 4 & 6 & 4 & 7 & 7 & 7 & 7 & 7 & 10 \\
\hline & 16 & 4 & 7 & 10 & 7 & 10 & 7 & 7 & 7 & 4 & 7 & & & 4 & 7 & 10 & 7 & 10 & 7 & 7 & 7 & 4 & 7 \\
\hline & 14 & 6 & 10 & 7 & 7 & 4 & 7 & 7 & 7 & 10 & 7 & & 14 & 6 & 10 & 7 & 7 & 4 & 7 & 7 & 7 & 10 & 7 \\
\hline & $\begin{array}{ll}0 & 10\end{array}$ & 4 & 7 & 7 & 7 & 4 & 9 & 7 & 6 & 6 & 10 & & $\begin{array}{ll}0 & 10\end{array}$ & 4 & 7 & 7 & 6 & 4 & 10 & 7 & 7 & 6 & 9 \\
\hline & $\begin{array}{ll}0 & 7\end{array}$ & 7 & 10 & 4 & 4 & 10 & 9 & 7 & 6 & 7 & 6 & & $\begin{array}{ll}0 & 7\end{array}$ & 7 & 10 & 4 & 4 & 9 & 6 & 9 & 9 & 6 & 6 \\
\hline & $\begin{array}{ll}0 & 7\end{array}$ & 7 & 7 & 7 & 9 & 9 & 3 & 6 & 4 & 9 & 9 & & $\begin{array}{ll}0 & 7\end{array}$ & 7 & 7 & 7 & 9 & 7 & 4 & 10 & 3 & 7 & 9 \\
\hline & $\begin{array}{ll}0 & 7\end{array}$ & 7 & 7 & 7 & 7 & 7 & 6 & 3 & 12 & 7 & 7 & & $\begin{array}{ll}0 & 7\end{array}$ & 7 & 7 & 7 & 9 & 7 & 4 & 3 & 10 & 7 & 9 \\
\hline & $\begin{array}{ll}0 & 7\end{array}$ & 7 & 7 & 7 & 6 & 6 & 4 & 12 & 9 & 6 & 6 & & $\begin{array}{ll}0 & 7\end{array}$ & 7 & 7 & 7 & 6 & 10 & 9 & 4 & 4 & 10 & 6 \\
\hline & $\begin{array}{ll}0 & 7\end{array}$ & 7 & 4 & 10 & 6 & 7 & 9 & 7 & 6 & 10 & 4 & & $\begin{array}{ll}0 & 7\end{array}$ & 7 & 4 & 10 & 6 & 6 & 6 & 9 & 9 & 9 & 4 \\
\hline & $\begin{array}{ll}0 & 4\end{array}$ & 10 & 7 & 7 & 10 & 6 & م & 7 & 6 & 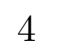 & 7 & & \pm & 10 & 7 & 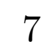 & 9 & 6 & 10 & 7 & 7 & 4 & 6 \\
\hline
\end{tabular}

\begin{tabular}{|c|c|c|c|c|c|c|c|c|c|c|c|c|c|c|c|c|c|c|c|c|c|c|c|}
\hline 53 & 119 & 10 & 10 & & & & & & & & & $\mathrm{~S} 4$ & & & & & & & & & & & \\
\hline & 119 & 19 & 19 & 19 & 0 & 0 & 0 & 0 & 0 & 0 & 0 & & 119 & $\begin{array}{ll}919 \\
\end{array}$ & 919 & 19 & 0 & 0 & 0 & 0 & 0 & 0 & 0 \\
\hline & 17 & 7 & 7 & 6 & 10 & 9 & 9 & 6 & 6 & 6 & 3 & & 17 & $7 \quad 7$ & 7 & 6 & 10 & 9 & 9 & 6 & 6 & 6 & 3 \\
\hline & 17 & 7 & 7 & 6 & 3 & 9 & 9 & 6 & 6 & 6 & 10 & & 17 & $7 \quad 7$ & 7 & 6 & 3 & 9 & 9 & 6 & 6 & 6 & 10 \\
\hline & & 7 & 4 & 9 & 9 & 6 & 4 & 9 & 6 & 6 & 9 & & 17 & $7 \quad 7$ & 4 & 9 & 9 & 6 & 4 & 9 & 6 & 6 & 9 \\
\hline & 16 & 6 & 9 & 6 & 6 & 4 & 6 & 7 & 10 & 10 & 6 & & 16 & 56 & 9 & 6 & 6 & 4 & 6 & 7 & 10 & 10 & 6 \\
\hline & $\begin{array}{ll}0 & 10\end{array}$ & 10 & 4 & 4 & 7 & 6 & 7 & 6 & 9 & 7 & 7 & & 010 & $\begin{array}{ll}0 & 9\end{array}$ & 6 & 3 & 6 & 7 & 6 & 10 & 7 & 7 & 6 \\
\hline & $\begin{array}{ll}0 & 10\end{array}$ & 3 & 6 & 9 & 6 & 9 & 6 & 6 & 7 & 9 & 6 & & $\begin{array}{ll}0 & 9\end{array}$ & 6 & 4 & 9 & 6 & 9 & 6 & 4 & 9 & 9 & 6 \\
\hline & $\begin{array}{ll}0 & 7\end{array}$ & 7 & 10 & 4 & 7 & 9 & 4 & 10 & 6 & 6 & 7 & & $\begin{array}{ll}0 & 9\end{array}$ & 9 & 10 & 6 & 9 & 7 & 6 & 6 & 6 & 6 & 9 \\
\hline & $\begin{array}{ll}0 & 7\end{array}$ & 7 & 7 & 7 & 7 & 4 & 10 & 9 & 3 & 9 & 7 & & $\begin{array}{ll}0 & 6\end{array}$ & 10 & 06 & 6 & 9 & 4 & 9 & 4 & 7 & 7 & 9 \\
\hline & $\begin{array}{ll}0 & 6\end{array}$ & 6 & 9 & 7 & 10 & 6 & 7 & 3 & 7 & 6 & 10 & & $\begin{array}{ll}0 & 6\end{array}$ & 6 & 7 & 9 & 6 & 6 & 9 & 9 & 10 & 3 & 6 \\
\hline & $\begin{array}{ll}0 & 6\end{array}$ & 6 & 7 & 9 & 6 & 6 & 9 & 9 & 10 & 3 & 6 & & $\begin{array}{ll}0 & 6\end{array}$ & 6 & 7 & 9 & 6 & 6 & 9 & 9 & 3 & 10 & 6 \\
\hline & $\begin{array}{ll}0 & 3\end{array}$ & 10 & 6 & 9 & 6 & 9 & 6 & 6 & 7 & 9 & 6 & & & 9 & 9 & 7 & 7 & 10 & 4 & 7 & 7 & 7 & 7 \\
\hline
\end{tabular}




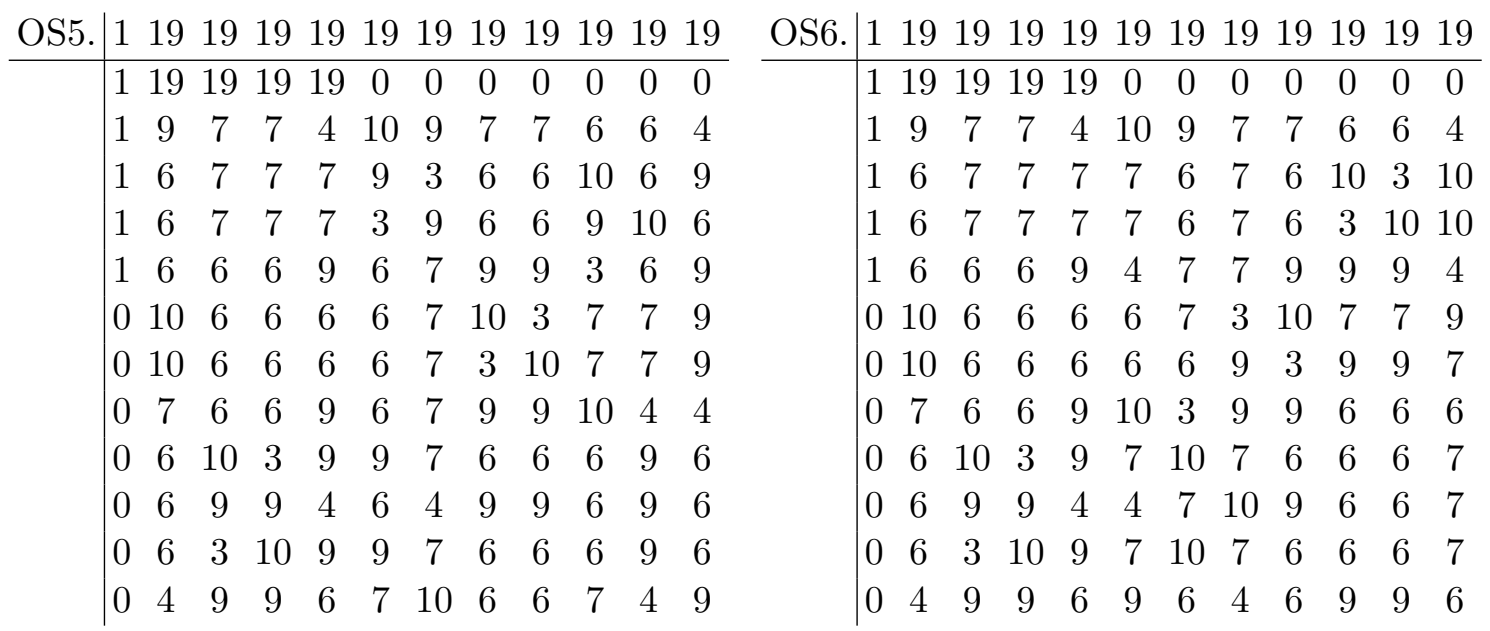

Remark 1. The actual indexing of these six orbit structures in order to produce an example is still an open problem.

\section{References}

[1] A. Beutelspacher. Einführung in die endliche Geometrie I. Bibliographisches Institut, Mannheim-Wien-Zürich, 1985.

[2] D. Crnković. Some new menon designs with parameters (196,91,42). Mathematical Communications, 10(2):169-175, 2005.

[3] V. Ćepulić. On symmetric block designs $(40,13,4)$ with automorphisms of order 5. Discrete Math., 28:45-60, 1994.

[4] M. Gashi. A construction of a symmetric design with parameters $(195,97,48)$ with help of frobenius group of order 4656. International Mathematical Forum, 5(8):383 $388,2009$.

[5] M. Gashi. On the symmetric block design with parameters $(306,61,12)$ admitting a group of order 61. European Journal of Pure and Applied Mathematics, 11(3):645-651, 2018.

[6] B. Huppert. Character Theory of Finite Groups. Walter de Gruyter - Berlin - New York, 1998.

[7] Z. Janko and Tran van Trung. Construction of a new symmetric block design for $(78,22,6)$ with help of tactical decompositions. J. Comb. Theory, Ser. A, 40:451$455,1995$. 
[8] T. Beth D. Jungnickel and H. Lenz. Design Theory. Bibliographisches Institut, Mannheim-Wien-Zürich, 1999.

[9] C. W. H. Lam. The search for a finite projective plane of order 10. Amer. Math. Monthly, 98:305-318, 1992.

[10] E. Lander. Symmetric designs: An algebraic approach. Cambridge University Press, 1983. 